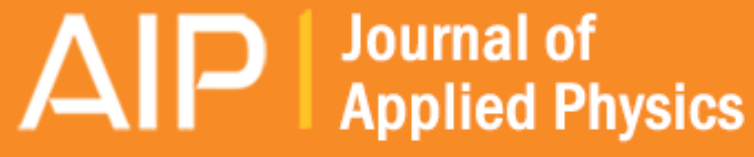

\section{Ge L3-edge x-ray absorption near-edge structure study of structural changes accompanying conductivity drift in the amorphous phase of Ge2Sb2Te5}

K. V. Mitrofanov, A. V. Kolobov, P. Fons, X. Wang, J. Tominaga, Y. Tamenori, T. Uruga, N. Ciocchini, and D. lelmini

Citation: Journal of Applied Physics 115, 173501 (2014); doi: 10.1063/1.4874415

View online: http://dx.doi.org/10.1063/1.4874415

View Table of Contents: http://scitation.aip.org/content/aip/journal/jap/115/17?ver=pdfcov

Published by the AIP Publishing

\section{Articles you may be interested in}

Publisher's Note: "Selective detection of tetrahedral units in amorphous GeTe-based phase change alloys using Ge L3-edge x-ray absorption near-edge structure spectroscopy" [Appl. Phys. Lett. 102, 111904 (2013)]

Appl. Phys. Lett. 102, 209901 (2013); 10.1063/1.4801918

Selective detection of tetrahedral units in amorphous GeTe-based phase change alloys using Ge L3-edge x-ray absorption near-edge structure spectroscopy

Appl. Phys. Lett. 102, 111904 (2013); 10.1063/1.4794870

Observation of polyamorphism in the phase change alloy Ge1Sb2Te4

Appl. Phys. Lett. 101, 151906 (2012); 10.1063/1.4759106

Characterization of local piezoelectric behavior of ferroelectric GeTe and Ge2Sb2Te5 thin films J. Appl. Phys. 112, 052018 (2012); 10.1063/1.4746087

Key experimental information on intermediate-range atomic structures in amorphous Ge2Sb2Te5 phase change material

J. Appl. Phys. 111, 083517 (2012); 10.1063/1.3703570

\section{$\underset{\substack{\text { Aubstrung } \\ \text { culs }}}{A}$ Re-register for Table of Content Alerts}

\section{Create a profile.}

\section{Sign up today!}




\title{
$\mathrm{Ge}_{3}$-edge x-ray absorption near-edge structure study of structural changes accompanying conductivity drift in the amorphous phase of $\mathrm{Ge}_{2} \mathrm{Sb}_{\mathbf{2}} \mathrm{Te}_{5}$
}

\author{
K. V. Mitrofanov, ${ }^{1}$ A. V. Kolobov, ${ }^{2, a)}$ P. Fons, ${ }^{2}$ X. Wang, ${ }^{3}$ J. Tominaga, ${ }^{3}$ Y. Tamenori, ${ }^{4}$ \\ T. Uruga, ${ }^{4}$ N. Ciocchini, ${ }^{5}$ and D. Ielmini ${ }^{5}$ \\ ${ }^{1}$ Nanoelectronics Research Institute, National Institute of Advanced Industrial Science and Technology (AIST), \\ 1-1-1 Higashi, Tsukuba 305-8562, Japan \\ ${ }^{2}$ Nanoelectronics Research Institute \& Green Nanoelectronics Center, National Institute of Advanced \\ Industrial Science and Technology (AIST), 1-1-1 Higashi, Tsukuba 305-8562, Japan and Synchrotron \\ Radiation Research Institute (JASRI), SPring-8, 1-1-1, Kouto, Sayo, Hyogo 679-5198, Japan \\ ${ }^{3}$ Nanoelectronics Research Institute \& Green Nanoelectronics Center, National Institute of Advanced \\ Industrial Science and Technology (AIST), 1-1-1 Higashi, Tsukuba 305-8562, Japan \\ ${ }^{4}$ Synchrotron Radiation Research Institute (JASRI), SPring-8, 1-1-1, Kouto, Sayo, Hyogo 679-5198, Japan \\ ${ }^{5}$ DEIB - Politecnico di Milano, Piazza L. Da Vinci 32, 20133 Milano, Italy
}

(Received 14 March 2014; accepted 21 April 2014; published online 2 May 2014)

\begin{abstract}
A gradual uncontrollable increase in the resistivity of the amorphous phase of phase-change alloys, such as $\mathrm{Ge}_{2} \mathrm{Sb}_{2} \mathrm{Te}_{5}$, known as drift, is a serious technological issue for application of phase-change memory. While it has been proposed that drift is related to structural relaxation, no direct structural

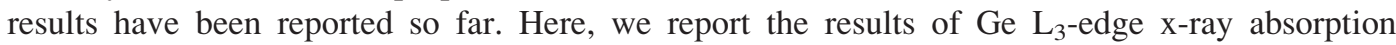
measurements that suggest that the drift in electrical conductivity is associated with the gradual conversion of tetrahedrally coordinated Ge sites into pyramidal sites, while the system still remains in the amorphous phase. Based on electronic configuration arguments, we propose that during this process, which is governed by the existence of lone-pair electrons, the concentration of free carriers in the system decreases resulting in an increase in resistance despite the structural relaxation towards the crystalline phase. (C) 2014 AIP Publishing LLC.

[http://dx.doi.org/10.1063/1.4874415]
\end{abstract}

\section{INTRODUCTION}

Phase-change materials, of which $\mathrm{Ge}_{2} \mathrm{Sb}_{2} \mathrm{Te}_{5}$ is one of the best performing representatives, are widely used in optical storage and also in electrical phase-change memory (PCM) devices. In the latter, the resistance is changed by applying current pulses that induce structural transitions between the crystalline (SET) and amorphous (RESET) states. The cell state is read by measuring the device resistance; hence, uncontrollable variation of the latter with time is undesirable. While the SET state is sufficiently stable, the RESET state that is formed by melting and rapidly quenching the phase-change alloy exhibits an increase in resistance with time. This uncontrollable change of resistance, limiting application of phase-change alloys in multilevel recording, is called drift and can be described by a power law

$$
R=R_{0}\left(t / t_{0}\right)^{\nu}
$$

where $R_{0}$ is the resistance at time $t_{0}$ and $\nu$ is called the drift exponent. The interested reader is referred to a recent review $^{1}$ or original publications $\mathrm{s}^{2,3}$ for more information.

Upon longer annealing times, the trend changes and the resistivity rapidly decreases by several orders of magnitude, which are associated with the establishment of long-range order.

Resistance drift is usually explained in terms of structural relaxation, a phenomenon well known for glasses and

\footnotetext{
a)a.kolobov@aist.go.jp
}

amorphous materials, which reduces the amount of structural defects such as wrong bonds, and vacancies. At the same time, it should be noted that structural relaxation towards the more ordered-in the limit crystalline-phase should typically result in a decrease in resistance, while experimentally the opposite change is observed at initial stages of annealing. This fact suggests that the relaxation process is more complicated in nature than just atomic ordering.

In a recent work, ${ }^{4}$ it was suggested that two different processes are responsible for the resistance change during the drift and crystallisation. In particular, the authors proposed that the increase in resistivity during the drift was associated with a change in the concentration of charge carriers as tetrahedral $\mathrm{Ge}$ sites are gradually transformed into pyramidal, or defective octahedral, sites, while an abrupt decrease in resistance upon crystallisation was caused by an increase in mobility due to establishment of long-range order. Below, in this work, the terms pyramidal and "defective octahedral" sites are used interchangeably. Originally, the term "defective octahedral" site was introduced ${ }^{5}$ to represent sites that had less than six (usually four) nearest neighbours located at distances up to $3.2 \AA$, while the term "pyramidal" was suggested ${ }^{6}$ to reflect the fact that $\mathrm{Ge}$ has short covalent bonds with only three of its neighbours.

In this work, we report on the results of $\mathrm{x}$-ray absorption fine structure (XAFS) measurements performed with the purpose of supporting or negating the proposed model. It has been previously demonstrated that Ge K-edge x-ray absorption near-edge structure (XANES) is sensitive to local structure about Ge species and can be effectively used to 
discriminate between the covalent (tetrahedral and/or pyramidal) configurations characteristic of the amorphous phase and resonant configurations characteristic of the crystalline phase. ${ }^{6}$ At the same time, differences between the tetrahedral and defective octahedral sites were rather small and establishment of a clear correlation between the XANES shape and the Ge site structure in the amorphous phases was not possible. On the other hand, Ge $\mathrm{L}_{3}$ XANES spectra in the amorphous phase demonstrated pronounced differences between the tetrahedral and pyramidal sites. ${ }^{7}$ Consequently, $\mathrm{Ge}_{3}$-edge XANES monitoring of the structural relaxation causing the resistance drift is a promising approach to unveil the origin of the latter.

\section{EXPERIMENTAL DETAILS}

Samples for the study were $200 \mathrm{~nm}$ thick $\mathrm{Ge}_{2} \mathrm{Sb}_{2} \mathrm{Te}_{5}$ layers deposited by magnetron sputtering onto oxidised $\mathrm{Si}$ wafers following the formation of TiN contacts with a $100 \mu \mathrm{m}$ gap between the contacts. The deposition was performed just before the experiment to preserve the as-deposited state. Measurements were done at SPring- 8 at beam line BL27SU. The existing facilities for the $\mathrm{L}_{3}$-edge measurements did not allow us to do in-situ annealing and the samples were pre-annealed at various conditions prior to the measurements. Annealing was performed in a $N_{2}$ atmosphere.

There are two ways to measure drift, one is to anneal the sample for a sufficiently long period of time at a fixed temperature and an alternative approach is to anneal the sample for a fixed duration of time at different temperatures. We have adopted the second approach. Samples were annealed for $24 \mathrm{~h}$ at temperatures ranging from 40 to $110^{\circ} \mathrm{C}$. Measurements were performed at room temperature immediately after the annealing (three different measurements were taken for each annealing temperature to ensure repeatability). Fig. 1 shows the resistance change caused by annealing. One can see that the resistance initially increases with annealing (the drift phenomenon proper) and then starts to decrease. The change in the trend is a prelude to crystallisation but since the sample resistance has the same order of magnitude (and even larger than) as the as-deposited sample, we consider this state to remain amorphous. Samples annealed in this way were subsequently used for the $\mathrm{L}_{3}$-edge measurements.

\section{RESULTS AND DISCUSSION}

We now proceed to the experimental XANES results. In Fig. 2, we show $\mathrm{Ge}_{3}$-edge spectra. A trend is clearly visible, namely, the step preceding the edge jump, that has been attributed to tetrahedrally coordinated Ge sites, is gradually decreasing (the onset shifts to higher energies decreasing the integral), suggesting a transformation of tetrahedral Ge sites into pyramidal (defective octahedral) sites. The monotonic change in the XANES spectra alongside with the reverse trend of the resistance change is in agreement with the proposed model. Indeed, the concentration of the pyramidal sites increases continuously, which accounts for the changes in XANES, while an establishment of resonant interaction and subsequent increase in carrier mobility upon their

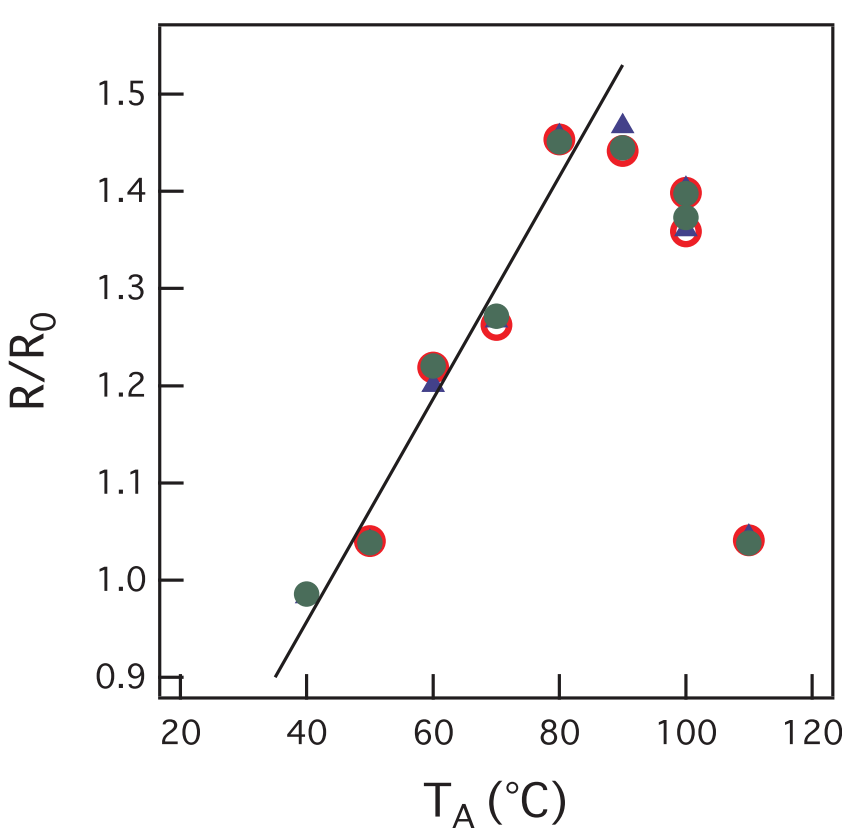

FIG. 1. Resistance drift: relative resistance after annealing for $24 \mathrm{~h}$ at $T_{A}$ measured at room temperature.

ordering, leads to a decrease in resistivity. Since the total resistance change during the drift per se results in an increase by about a factor of two and the decrease in resistivity due to crystallisation is more than two orders of magnitude, it is not surprising that the formation of just a few resonantly bonded sites that involve pyramidal configurations can induce a measurable change in resistance.

The conversion between the tetrahedral sites and octahedral sites is possible due to the dual bonding nature between $\mathrm{Ge}$ and $\mathrm{Te}$ atoms in amorphous GeTe and related alloys such as $\mathrm{Ge}_{2} \mathrm{Sb}_{2} \mathrm{Te}_{5}$ and is shown schematically in Fig. 3. Ge atoms generally prefer to be $s p^{3}$ hybridized and tetrahedrally coordinated, when forming covalent bonds with its neighbours, satisfying the Mott $8-\mathrm{N}$ rule. At the same time, in the crystalline state of $\mathrm{GeTe}$, due to establishment of resonant three-center two-electron bonds, ${ }^{8}$ where each atom is bonded

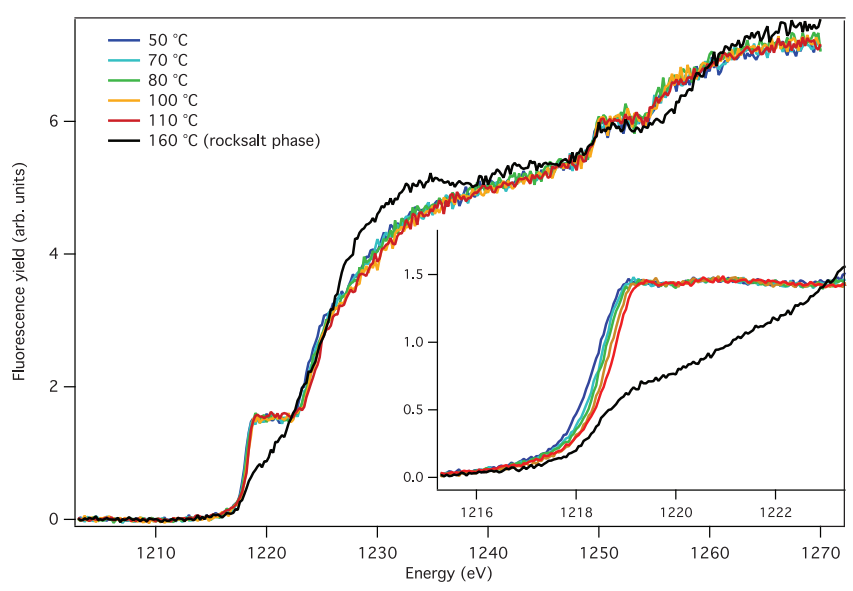

FIG. 2. A series of $\mathrm{Ge}_{3}$-edge spectra measured at room temperature for samples pre-annealed for $24 \mathrm{~h}$ at different temperatures (marked in the figure) one day before the measurement. The inset shows a zoomed-in pre-edge region, where the onset of the step-like features clearly shifts to higher energies for higher annealing temperatures, decreasing the integral. 

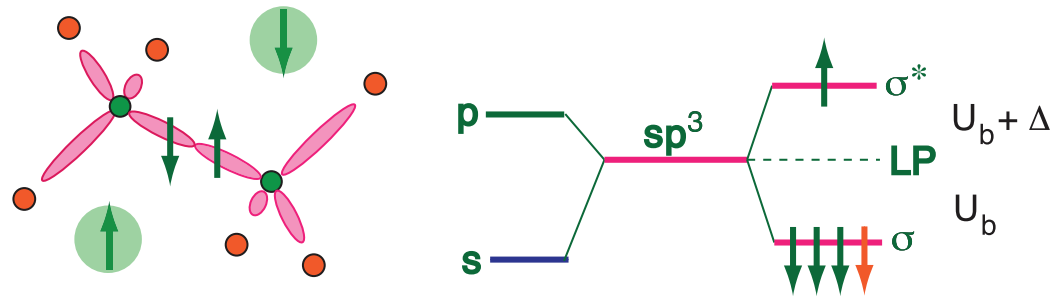

$\int_{0}^{0}$
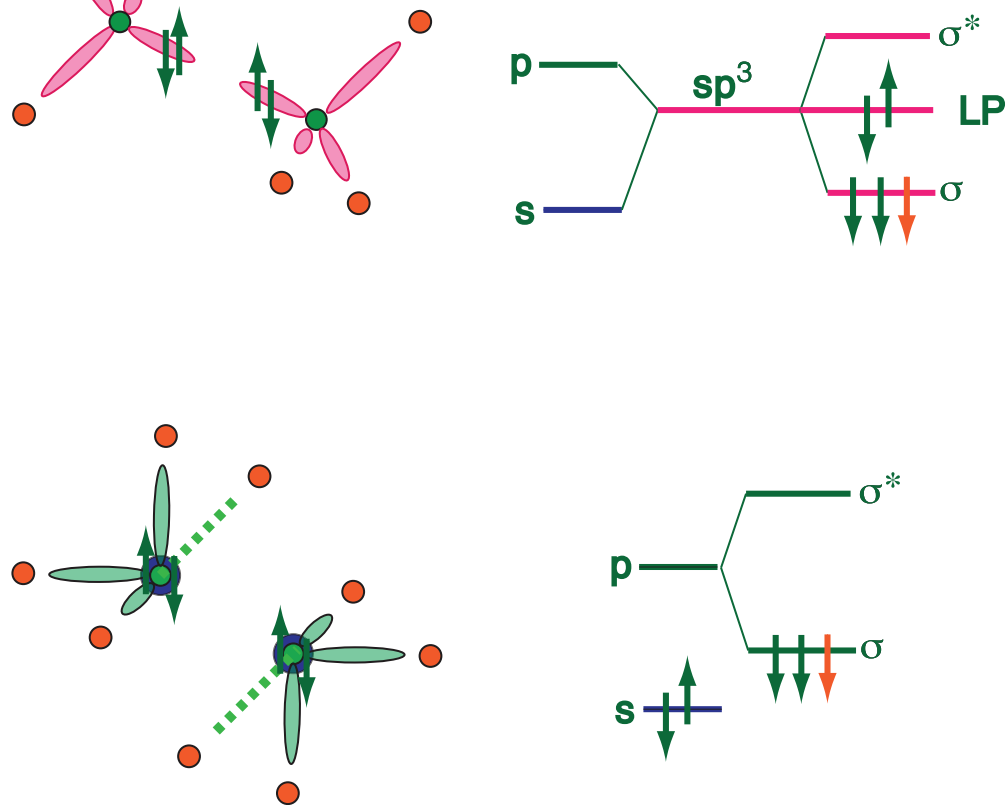

FIG. 3. Transformation between a pair of pyramidally bonded Ge sites and tetrahedrally bonded sites (panels from bottom to top), the atomic configurations are shown on the left and the corresponding electronic diagrams are shown on the right. Ge atoms and valence electrons originating from them are shown in green, Te atoms and the electron donated by $\mathrm{Te}$ atoms to form a dative bond are shown in orange. Additionally, in the diagrams, $s$ orbitals are shown in blue, $p$ orbitals are in green, and $s p^{3}$ orbitals are in magenta. In the crystalline phase, due to resonant bonding, pyramidal $p$ bonded configurations are formed, preserving the lone-pair on the $s$ orbital (bottom panel). Provided the relaxation during the amorphization process increases the valence angles, $s p^{3}$-hybridization is promoted and the lone-pair finds itself on an $s p^{3}$-orbital (middle panel). The formation of a $\mathrm{Ge}-\mathrm{Ge}$ bond (upper panel) consumes one electron from each lone-pair, promoting the other one to the anti-bonding orbital (conduction band). Since $\Delta \ll U_{b}$, this process does not cost much energy. to its first nearest neighbours on both sides using the same p-orbital of Ge (Refs. 9-11), the total energy becomes lower and it is energetically more favourable for Ge atoms to preserve their unhybridized atomic electronic configuration $s^{2} p_{x}^{1} p_{y}^{1} p_{z}^{0}$ and accept one electron from a Te lone-pair orbital to form one of the three covalent bonds, one being a dative bond in this case. The details of this process have been discussed in detail earlier. ${ }^{8,12,13}$ The gain in energy arising due to the formation of the three extra (resonant) bonds accounts for the cubic-like structure of GeTe and related alloys in the crystalline phase.

Resonant bonds are partially preserved in the amorphous phase, as is evidenced by the existence of so-called defective octahedral sites when Ge atoms have four (or five) neighbours with three of them located at a typical Ge-Te covalent distance of around $2.6 \AA$ and others at a distance close to 3.1-3.2 $\AA$, i.e., the same distance as the longer Ge-Te bond in the crystalline phase. Depending on the details of the structure, such configurations may be energetically favored over the tetrahedral sites as evidenced by numerous ab-initio studies. ${ }^{5,14,15}$ At the same time, even if the bonding angles in this configuration are close to $90^{\circ}$, the bonding is not purely p-type. $s p^{3}$ hybridisation for such sites has been reported earlier ${ }^{14}$ and, subsequently, an electron localisation function analysis revealed that the lone-pair electrons are actually located on an $s p^{3}$ orbital of Ge. ${ }^{4}$ The situation is not unusual and the presence of lone-pair electrons on hybridised orbitals is not unknown (see, e.g., Ref. 16). The degree of hybridization is influenced by the local atomic structure. Thus, while in a water molecule the $\mathrm{O}$ atom uses two of its $p$-orbitals to form two $\sigma$ bonds with hydrogen, ${ }^{17}$ electrostatic repulsion between the hydrogen atoms (charged due to the electronegativity difference between hydrogen and oxygen atoms) makes the bonding angle close to $109^{\circ}$, which promotes $s p^{3}$ hybridization. In several recent textbooks, ${ }^{16,18,19}$ the oxygen atom in a water molecule is described as $s p^{3}$ hybridized with two of its $s p^{3}$ orbitals occupied by lone-pair electrons, although the underlying cause for the hybridization was not addressed. Similarly, as the structure relaxes and the valence angles deviate from $90^{\circ}$ (e.g., increase), the degree of hybridization changes and the lone-pair electrons, subtended at the $s$-orbital in pyramidal sites, may move into $s p^{3}$ orbitals making it possible to form an additional bond.

It should also be noted that tetrahedrally bonded $\mathrm{Ge}$ atoms were found to have at least one wrong bond such as a Ge-Ge bond. ${ }^{5}$ The presence of Ge-Ge bonds and tetrahedral sites in the amorphous (as-deposited) phase has been also confirmed by EXAFS, ${ }^{20}$ with their concentration in the reamorphized phase obtained from the crystalline phase being much lower. ${ }^{6,21}$ The result is not surprising since the 
as-deposited phase is formed under highly non-equilibrium conditions where local energy gain due to $s p^{3}$ hybridization dominates. It is thus likely that the tetrahedral sites are formed when two Ge atoms approach each other during the amorphization process.

One can thus view two different electronic configurations of Ge atoms in the amorphous phase (Fig. 3), namely, the one with the lone-pair electrons strongly localised on (almost unhybridized) Ge atoms in pyramidal sites with octahedral bonding angles and the other one with loosely bonded lone-pair electrons in three-fold coordinated atoms with tetrahedral bonding angles. ${ }^{22}$ In the latter case, promotion of one of the electrons into the anti-bonding state (conduction band) leaves behind an unpaired electron that can serve to form an additional bond with gain in energy due to subsequent lattice relaxation serving to stabilize the structure. This process generates additional charge carriers in the system. It is important that these-essentially non-equilibriumcharge carriers cannot recombine because once the bonds are formed, the original non-bonding (LP) states disappear. This process is similar to the generation of persistent photoconductivity in chalcogenide glasses at low temperature, ${ }^{24}$ attributed to the formation of additional bonds under photoexcitation. $^{25}$

Upon annealing, the reverse process is taking place (Fig. 3 , top to bottom). Namely, Ge-Ge bonds between tetrahedrally coordinated atoms break, leaving behind two dangling bonds. The latter can trap electrons decreasing the concentration of available free charge carriers. Subsequent lattice relaxation towards octahedral bonding angles results in deepening of the lone-pair electron orbital decreasing the degree of $s p^{3}$ hybridization, in the limit, the bonding is purely between the $p$ orbitals and the lone-pair is located on the $s$ orbital.

This model provides a clear explanation as to why atomic relaxation towards the crystalline phase is accompanied by an initial increase in resistance. It also provides an explanation why pre-annealing leads to a significant increase of the crystallisation rate. ${ }^{23}$ Indeed, if pre-annealing is associated with destruction of the tetrahedral sites, subsequent ordering of pyramidal sites into a cubic lattice can proceed much faster.

In summary, evolution of the $\mathrm{L}_{3}$-edge XANES spectra are indicative of the gradual transformation of tetrahedral $\mathrm{Ge}$ sites into pyramidal Ge sites in the amorphous phase prior to the onset of crystallisation. This transformation, accompanied by a change in the number of electrons involved in bond formation, that is possible in GeTe-based alloys due to the presence of lone-pair electrons, is proposed to account for the observed increase in resistance (drift) during the relaxation process. Subsequent ordering of the pyramidal sites through the establishment of resonant bonding generates the crystalline phase.

\section{ACKNOWLEDGMENTS}

XANES measurements were performed at SPring-8 within the 2013A1200 and 2013A1449 proposals. Work at Politecnico di Milano was supported in part by Fondazione Cariplo under Grant No. 2010-0500.

${ }^{1}$ D. Ielmini, in Nonvolatile Memories: Materials, Devices and Applications, edited by T.-Y. Tseng and S. M. Sze (American Scientific Publishers, 2012), Vol. 2, pp. 1-27.

${ }^{2}$ A. Pirovano, A. L. Lacaita, F. Pellizzer, S. A. Kostylev, A. Benvenuti, and R. Bez, IEEE Trans. Electron Devices 51, 714 (2004).

${ }^{3}$ D. Ielmini, A. L. Lacaita, and D. Mantegazza, IEEE Trans. Electron Devices 54, 308 (2007).

${ }^{4}$ A. V. Kolobov, P. Fons, and J. Tominaga, Phys. Rev. B 87, 155204 (2013).

${ }^{5}$ S. Caravati, M. Bernasconi, T. Kühne, M. Krack, and M. Parrinello, Appl. Phys. Lett. 91, 171906 (2007).

${ }^{6}$ M. Krbal, A. V. Kolobov, P. Fons, J. Tominaga, S. R. Elliott, J. Hegedus, and T. Uruga, Phys. Rev. B 83, 054203 (2011).

${ }^{7}$ M. Krbal, A. V. Kolobov, P. Fons, K. V. Mitrofanov, Y. Tamenori, J. Hegedüs, S. R. Elliott, and J. Tominaga, Appl. Phys. Lett. 102, 111904 (2013).

${ }^{8}$ A. V. Kolobov, M. Krbal, P. Fons, J. Tominaga, and T. Uruga, Nat. Chem. 3, 311 (2011).

${ }^{9}$ G. Lucovsky and R. White, Phys. Rev. B 8, 660 (1973).

${ }^{10}$ J. Robertson, K. Xiong, and P. W. Peacock, Thin Solid Films 515, 7538 (2007).

${ }^{11}$ K. Shportko, S. Kremers, M. Woda, D. Lencer, J. Robertson, and M. Wuttig, Nat. Mater. 7, 653 (2008).

${ }^{12}$ M. Xu, Y. Cheng, H. Sheng, and E. Ma, Phys. Rev. Lett. 103, 195502 (2009).

${ }^{13}$ A. V. Kolobov, P. Fons, and J. Tominaga, Phys. Status Solidi B 249, 1902 (2012).

${ }^{14}$ J. Akola and R. Jones, Phys. Rev. B 76, 235201 (2007).

${ }^{15}$ J. Hegedüs and S. Elliott, Nat. Mater. 7, 399 (2008).

${ }^{16}$ W. L. Masterton, C. N. Hurley, and E. J. Neth, Chemistry: Principles and Reactions, 7th ed. (Cengage Learning, 2011).

${ }^{17}$ P. W. Atkins, Physical Chemistry, 6th ed. (Oxford University Press, 1998).

${ }^{18}$ D. W. Oxtoby, H. P. Gillis, and A. Campion, Principles of Modern Chemistry (Cengage Learning, 2011).

${ }^{19}$ D. D. Ebbing and S. D. Gammon, General Chemistry (Cengage Learning, 2009).

${ }^{20}$ D. Baker, M. Paesler, G. Lucovsky, S. Agarwal, and P. Taylor, Phys. Rev. Lett. 96, 255501 (2006).

${ }^{21}$ J. Akola, J. Larrucea, and R. O. Jones, Phys. Rev. B 83, 094113 (2011).

${ }^{22}$ A. V. Kolobov, P. Fons, M. Krbal, and J. Tominaga, Phys. Status Solidi A 209, 1031 (2012).

${ }^{23}$ D. Loke, T. H. Lee, W. Wang, L. P. Shi, R. Zhao, Y. C. Yeo, T. C. Chong, and S. Elliott, Science 336, 1566 (2012).

${ }^{24}$ N. Toyosawa and K. Tanaka, Phys. Rev. B 56, 7416 (1997).

${ }^{25}$ A. Kolobov, H. Oyanagi, K. Tanaka, and K. Tanaka, Phys. Rev. B 55, 726 (1997). 